Mathematical Modelling and Analysis

Volume 22 Number 3, May 2017, 300-310

https://doi.org/10.3846/13926292.2017.1307790

(c) Vilnius Gediminas Technical University, 2017
Publisher: Taylor\&Francis and VGTU

http://www.tandfonline.com/TMMA

ISSN: $1392-6292$

eISSN: $1648-3510$

\title{
Two-Scale Estimates for Special Finite Discrete Operators*
}

\author{
Alexander V. Vasilyev ${ }^{a, b}$ and Vladimir B. Vasilyev ${ }^{a, b}$ \\ ${ }^{a}$ Belgorod National Research University \\ Studencheskaya 14/1, 308007 Belgorod, Russia \\ ${ }^{b}$ Lipetsk State Technical University \\ E-mail(corresp.): vladimir.b.vasilyev@gmail.com \\ E-mail: alexvassel@gmail.com
}

Received October 9, 2016; revised March 4, 2017; published online May 15, 2017

Abstract. We consider a certain finite discrete approximation for multidimensional Calderon-Zygmund integral operator and give a comparison between solutions of corresponding equations in some spaces of discrete functions.

Keywords: finite discrete operator, Calderon-Zygmund operator, error estimate.

AMS Subject Classification: 42B20; 65R20.

\section{Introduction}

We consider the following Calderon-Zygmund singular integral operator [10]

$$
(K u)(x) \equiv v \cdot p \cdot \int_{\mathbb{R}^{m}} K(x-y) u(y) d y, \quad x \in \mathbb{R}^{m},
$$

in the Lebesgue space $L_{2}\left(\mathbb{R}^{m}\right)$ and its discrete analogue of the following type

$$
\left(K_{d} u_{d}\right)(\tilde{x}) \equiv \sum_{\tilde{y} \in h \mathbb{Z}^{m}} K_{d}(\tilde{x}-\tilde{y}) u_{d}(\tilde{y}) h^{m}, \quad \tilde{x} \in h \mathbb{Z}^{m}
$$

in the space $L_{2}\left(h \mathbb{Z}^{m}\right) \equiv l^{2}$ of functions $u_{d}$ of a discrete variable $\tilde{x} \in h \mathbb{Z}^{m}$.

We recall [10] that that a symbol $\sigma(\xi)$ of the operator $K$ is the Fourier transform of its kernel in principal value sense

$$
\sigma(\xi)=\lim _{\varepsilon \rightarrow 0, N \rightarrow+\infty} \int_{\varepsilon<|x|<N} K(x) e^{i x \cdot \xi} d x,
$$

* This work was supported by RFBR and Lipetsk regional government of Russia, grant No. 14-41-03595-r-center-a 
and such symbol is called an elliptic symbol if

$$
\inf _{\xi}|\sigma(\xi)|>0
$$

Our main goal is the following. Starting from the operator (1.1) we introduce the discrete operator (1.2) acting in infinite dimensional space so that it preserves basic properties of the operator (1.1) related to an ellipticity and invertibility $[18,19,20,21,22]$. Further to obtain computational algorithms we would like to construct finite dimensional analogue of the operator (1.2) preserving same properties, and to obtain results on comparison of these operators and solutions of corresponding equations.

\subsection{Some previous approaches and studies}

In books $[2,3,5,6,14,15,17]$ authors present studies on convolution equations, one-dimensional singular integral equations and multidimensional weakly singular integral equations related to approximate solution of these equations.

As usual there are the following questions in these studies: 1) to find an approximate equation desirable in a finite-dimensional ( $N$-dimensional) space so that under enough large $N$ this approximate equation will be uniquely solvable in an appropriate space; 2) to obtain an error estimate between exact solution of an initial equation and exact solution of approximate equation in dependence on $N$. As far as we know these questions were investigated fully for one-dimensional singular integral equations on smooth curves $L$ in a complex plane $\mathbb{C}[8,15]$

$$
a(t) u(t)+\frac{b(t)}{\pi i} v \cdot p \cdot \int_{L} \frac{u(\tau)}{t-\tau} d \tau=v(t), \quad t \in L,
$$

convolution equations with integrable kernel $K(x)$ on a straight line

$$
a(x) u(x)+\int_{-\infty}^{+\infty} K(x-y) u(y) d y=v(x), \quad x \in \mathbb{R},
$$

and some their multidimensional analogues $[2,3,5]$ and for multidimensional integral equations with a weak singularity

$$
a(x) u(x)+\int_{D} K(x, y) u(y) d y=v(x), \quad x \in D \subset \mathbb{R}^{m},
$$

where $D$ is a domain with a smooth boundary $\partial D$ and the kernel $K(x, y)$ satisfies the estimate

$$
|K(x, y)| \leq \frac{c}{|x-y|^{m-\alpha}}, \quad 0<\alpha \leq 1
$$

last equations are generated by compact integral operators in appropriate spaces [17].

An algebraic approach based on a local principle for studying certain finite approximations for integral operators was used in many papers, and its 
development for last years is presented in books for example $[2,3,6]$. But this method of $C^{*}$-algebras permits to prove a solvability of approximating equation but it can't help for obtaining an error estimate for solutions of these equations. Moreover concrete applications of the method are related as a rule to one-dimensional singular integral operators and equations.

For an error estimate there are some computational results $[9,12,13,16]$, and a lot of results are related to special equations for applied problems (see for example [7]). Multidimensional case is more complicated because there is no such advanced theory similar classical Riemann boundary value problem $[4,11]$.

We would like to note that in general there are no valuable results for approximate solution of multidimensional singular integral equations with Calderon - Zygmund operators although such equations in distinct form arise in many problems of partial differential equations and mathematical physics [10]. In our opinion a direct digitization is more convenient for computer calculations than other methods, and we try to use and justify this approach.

\section{Discrete spaces and transformations}

\subsection{Definitions and notations}

We accept the following conventions and notations. The kernel $K(x)$ is a differentiable function on the unit sphere in $\mathbb{R}^{m}, K(0) \equiv 0$, and $K_{d}$ is a restriction of the kernel $K$ on lattice points $h \mathbb{Z}^{m}$. Let $h$ be a size of a "spatial quant", $N$ be a size of our "Universe". These parameters will be tend to zero and infinity respectively. For the operator (1.1) we'll use the following reduction. First we replace the operator (1.1) by the discrete operator (1.2), and second we approximate this series by a special finite sum

$$
\sum_{\tilde{y} \in h \mathbb{Z}^{m} \cap Q_{N}} K_{d, N}(\tilde{x}-\tilde{y}) u_{d}(\tilde{y}) h^{m}, \quad \tilde{x} \in h \mathbb{Z}^{m} \cap Q_{N} .
$$

For $K_{d, N}$ in the formula (2.1) we suggest a following construction. If $K_{d}$ is a restriction of the continual kernel $K$ on lattice points $h \mathbb{Z}^{m}$ then we take a restriction of the $K_{d}$ on points $h \mathbb{Z}^{m} \cap Q_{N}$, where

$$
Q_{N}=\left\{x \in \mathbb{R}^{m}: x=\left(x_{1}, \cdots, x_{m}\right), \max _{1 \leq k \leq m}\left|x_{k}\right| \leq N\right\}
$$

and denote by $K_{d, N}$ its periodic continuation on the whole $h \mathbb{Z}^{m}$.

We would like to justify a following sequence of transformations, "continual" operator $(1.1) \longrightarrow$ "infinite discrete" operator $(1.2) \longrightarrow$ "finite discrete" operator (2.1) with corresponding estimates on $h$ and $N$. The comparison of (1.1) and (1.2) was given in papers' series [18,19,20,21,22], and here we consider a comparison between (1.2) and (2.1).

Let's denote by $P_{N}$ the restriction operator $h \mathbb{Z}^{m} \longrightarrow h \mathbb{Z}^{m} \cap Q_{N}$, and the space $L_{2}\left(h \mathbb{Z}^{m} \cap Q_{N}\right)$ is denoted by $l_{N}^{2}$ so that $P_{N}$ is a projector $l^{2} \rightarrow l_{N}^{2}$.

Definition 1. Approximation rate of operators $K_{d}$ and $K_{d, N}$ is called the following operator norm

$$
\left\|K_{d, N} P_{N}-P_{N} K_{d}\right\|_{l^{2} \rightarrow l_{N}^{2}}
$$


It is non-trivial to obtain an estimate for the operator norm, but we'll give an estimate for an individual element assuming the existence of some its properties. More precisely we'll suppose that the element $u_{d}$ is a restriction of the function $u$ which has Hölder property in $\mathbb{R}^{m}$ and $u(x)=o\left(|x|^{\gamma}\right),|x| \rightarrow \infty$, with some $\gamma>0$.

We will obtain a "weak estimate" for approximation rate but enough for our purposes. We assume additionally that a function $u_{d}$ is a restriction on $h \mathbb{Z}^{m}$ of continuous function with certain estimates [20,21]. Let's define the discrete space $C_{h}(\alpha, \beta)$ as a functional space of discrete variable $\tilde{x} \in h \mathbb{Z}^{m}$ with finite norm

$$
\left\|u_{d}\right\|_{C_{h}(\alpha, \beta)}=\left\|u_{d}\right\|_{C_{h}}+\sup _{\tilde{x}, \tilde{y} \in h \mathbb{Z}^{m}} \frac{|\tilde{x}-\tilde{y}|^{\alpha}}{(\max \{1+|\tilde{x}|, 1+|\tilde{y}|\})^{\beta}} .
$$

It means that the function $u_{d} \in C_{h}(\alpha, \beta)$ satisfies the following estimates

$$
\begin{aligned}
& \left|u_{d}(\tilde{x})-u_{d}(\tilde{y})\right| \leq c \frac{|\tilde{x}-\tilde{y}|^{\alpha}}{(\max \{1+|\tilde{x}|, 1+|\tilde{y}|\})^{\beta}}, \\
& \left|u_{d}(\tilde{x})\right| \leq \frac{c}{(1+|\tilde{x}|)^{\beta-\alpha}}, \quad \forall \tilde{x}, \tilde{y} \in h \mathbb{Z}^{m}, \alpha, \beta-\alpha>m, 0<\alpha<1 .
\end{aligned}
$$

Let us note that under required assumptions $C_{h}(\alpha, \beta) \subset L_{2}\left(h \mathbb{Z}^{m}\right)$, and these discrete space are discrete analogue of corresponding subspaces of continuous functions [1].

Theorem 1. For operators $K_{d}$ and $K_{d, N}$ the following estimate

$$
\left\|K_{d, N} P_{N} u_{d}-P_{N} K_{d} u_{d}\right\|_{l_{N}^{2}} \leq C N^{m+2(\alpha-\beta)}
$$

holds, where constant $C$ doesn't depend on $N, h$.

Proof. Let us write

$$
\left(P_{N} K_{d}-K_{d, N} P_{N}\right) u_{d}=P_{N} K_{d} P_{N} u_{d}-K_{d, N} P_{N} u_{d}+P_{N} K_{d}\left(I-P_{N}\right) u_{d},
$$

where $I$ is an identity operator in $L_{2}\left(h \mathbb{Z}^{m}\right)$.

First two summands have annihilated, and we need to estimate only the last summand. We have

$$
\left\|P_{N} K_{d}\left(I-P_{N}\right) u_{d}\right\| \leq C\left\|\left(I-P_{N}\right) u_{d}\right\|
$$

because norms of operators $K_{d}$ are uniformly bounded, and for the last norm taking into account above estimates we can write

$$
\left\|\left(I-P_{N}\right) u_{d}\right\|^{2} \leq C \sum_{\tilde{x} \in h \mathbb{Z}^{m} \backslash Q_{N}}\left|u_{d}(\tilde{x})\right|^{2} h^{m} \leq C \sum_{\tilde{x} \in h \mathbb{Z}^{m} \backslash Q_{N}} \frac{h^{m}}{(1+|\tilde{x}|)^{2(\beta-\alpha)}} \leq
$$

and further

$$
C \int_{\mathbb{R}^{m} \backslash Q_{N}}|x|^{2(\alpha-\beta)} d x .
$$

The last integral using spherical coordinates gives the estimate $N^{m+2(\alpha-\beta)}$ which tends to 0 under $n \rightarrow \infty$ if $\beta>\alpha+m / 2$. 
Remark 1. Similar theorem was obtained in $[19,20]$ for the space $C_{h}(\alpha, \beta)$.

This theorem plays a key role for obtaining an estimate for approximate solution of an multidimensional singular integral equation with the operator (1.1) and permits to use fast Fourier transform for evaluating a numerical solution. Some test calculations were given in [20].

\subsection{Discrete Fourier transform}

We define the discrete Fourier transform for a function $u_{d}$ of a discrete variable $\tilde{x} \in h \mathbb{Z}^{m}$ as the series

$$
\tilde{u}_{d}(\xi)=\sum_{\tilde{x} \in h \mathbb{Z}^{m}} e^{-i x \cdot \xi} u_{d}(\tilde{x}) h^{m}, \quad \xi \in \hbar \mathbb{T}^{m},
$$

where $\hbar=h^{-1} /(2 \pi)$.

This discrete Fourier transform has same properties like standard continual Fourier transform, particularly for a discrete convolution of two discrete functions $u_{d}, v_{d}$

$$
\left(u_{d} * v_{d}\right)(\tilde{x}) \equiv \sum_{\tilde{y} \in h \mathbb{Z}^{m}} u_{d}(\tilde{x}-\tilde{y}) v_{d}(\tilde{y}) h^{m}
$$

we have the well known multiplication property

$$
\left(F_{d}\left(u_{d} * v_{d}\right)\right)(\xi)=\left(F_{d} u_{d}\right)(\xi) \cdot\left(F_{d} v_{d}\right)(\xi) .
$$

If we apply this property to the operator $K_{d}$ we obtain

$$
\left(F_{d}\left(K_{d} u_{d}\right)\right)(\xi)=\left(F_{d} K_{d}\right)(\xi) \cdot\left(F_{d} u_{d}\right)(\xi) .
$$

Let us denote $\left(F_{d} K_{d}\right)(\xi) \equiv \sigma_{d}(\xi)$ and give the following

Definition 2. The function $\sigma_{d}(\xi), \xi \in \hbar \mathbb{T}^{m}$, is called a periodic symbol of the operator $K_{d}$.

We will assume below that the symbol $\sigma_{d}(\xi) \in C\left(\hbar \mathbb{T}^{m}\right)$ therefore we have immediately the following

Property 1. The operator $K_{d}$ is invertible in the space $L_{2}\left(h \mathbb{Z}^{m}\right)$ iff $\sigma_{d}(\xi) \neq$ $0, \forall \xi \in \hbar \mathbb{T}^{m}$.

Definition 3. A continuous periodic symbol is called an elliptic symbol if $\sigma_{d}(\xi) \neq 0, \forall \xi \in \hbar \mathbb{T}^{m}$.

So we see that an arbitrary elliptic periodic symbol $\sigma_{d}(\xi)$ corresponds to an invertible operator $K_{d}$ in the space $L_{2}\left(h \mathbb{Z}^{m}\right)$.

Remark 2. It was proved earlier that operators (1.1) and (1.2) for cases $D=$ $\mathbb{R}^{m}, D=\mathbb{R}_{+}^{m}$ are invertible or non-invertible in spaces $L_{2}\left(\mathbb{R}^{m}\right), L_{2}\left(\mathbb{R}_{+}^{m}\right)$ and $L_{2}\left(h \mathbb{Z}^{m}\right), L_{2}\left(h \mathbb{Z}_{+}^{m}\right)$ simultaneously $[18,22]$. 


\subsection{Finite discrete Fourier transform}

Definition 4. For a function $u_{d, N} \in l_{N}^{2}$ its finite discrete Fourier transform is defined by the formula

$$
\tilde{u}_{d, N}(\tilde{\xi})=\sum_{\tilde{x} \in h \mathbb{Z}^{m} \cap Q_{N}} e^{i \tilde{x} \cdot \tilde{\xi}} u_{d, N}(\tilde{x}) h^{m}, \quad \tilde{\xi} \in \hbar \mathbb{Z}^{m} \cap Q_{N} .
$$

Definition 5. A symbol of the operator $K_{d, N}$ is called the function $\sigma_{d, N}(\tilde{\xi})$ of a discrete variable $\tilde{\xi} \in \hbar \mathbb{Z}^{m} \cap Q_{N}$ defined by the formula

$$
\sigma_{d, N}(\tilde{\xi})=\sum_{\tilde{x} \in h \mathbb{Z}^{m} \cap Q_{N}} e^{i \tilde{x} \cdot \tilde{\xi}} K_{d, N}(\tilde{x}) h^{m}, \quad \tilde{\xi} \in \hbar \mathbb{Z}^{m} \cap Q_{N} .
$$

\section{Comparison between infinite and finite discrete opera- tors}

Theorem 2. If the operator $K_{d}$ is invertible in the space $l^{2}$ then the operator $K_{d, N}$ is invertible in the space $l_{N}^{2}$ for enough large $N$.

Proof. Let the function

$$
\sum_{\tilde{x} \in Q_{N}^{d}} K_{d, N}(\tilde{x}) e^{i \tilde{x} \cdot \xi} h^{m}, \quad \xi \in \hbar \mathbb{T}^{m}
$$

is a segment of the Fourier series

$$
\sum_{\tilde{x} \in h \mathbb{Z}^{m}} K_{d}(\tilde{x}) e^{i \tilde{x} \cdot \xi} h^{m}, \quad \xi \in \hbar \mathbb{T}^{m}
$$

and according our assumptions this is continuous function on $\hbar \mathbb{T}^{m}$. Therefore values of the partial sum coincide with values of $\sigma_{d, N}$ in points $\tilde{\xi} \in R_{N}^{d} \equiv$ $\hbar \mathbb{Z}^{m} \cap Q_{N}$. Besides these partial sums are continuous functions on $\hbar \mathbb{T}^{m}$.

\subsection{Some auxiliary results}

Lemma 1. The norm of operator $K_{d} ; l^{2} \rightarrow l^{2}$ doesn't depend on $h$

For the proof see [19].

Lemma 2. The norm of operator $K_{d, N}: l_{N}^{2} \rightarrow l_{N}^{2}$ doesn't depend on $N, h$.

Proof. Using the Theorem 2 and the property that the norm of the operator $K_{d, N}$ is equivalent to $\max _{\xi \in \hbar \mathbb{Z}^{m} \cap Q_{N}}\left|\sigma_{d, N}(\xi)\right|$ (see also [10]) we obtain the required assertion. 


\section{Correlation between $N, h$ and location of $\tilde{x}$}

The proved Theorem 1 is a very rough estimate. It is possible to obtain more exact error estimate for finite discrete solution taking into account a location of the point $\tilde{x}$ and relations between parameters $N$ and $h$.

We consider three types of equations

$$
\begin{aligned}
& K u=v, \\
& K_{d} u_{d}=P_{d} v \equiv v_{d},
\end{aligned}
$$

where $P_{d}$ is a restriction operator which given continuous function $v$ defined on $\mathbb{R}^{m}$ maps to a collection of its values on $\mathbb{Z}^{m}$, and

$$
K_{d, N} u_{d, N}=v_{d, N} \equiv P_{N} v_{d}
$$

and would like to have an estimate for nearness of their solutions.

Let us denote by $r(\tilde{x})$ the distance between $\tilde{x} \in h \mathbb{Z}^{m} \cap Q_{N}$.

Theorem 3. Let $v_{d} \in C_{h}(\alpha, \beta)$. Then $\forall \tilde{x} \in \mathbb{Z}^{m} \cap Q_{N}$ the following estimate

$$
\left|u_{d}(\tilde{x})-u_{d, N}(\tilde{x})\right| \leq c_{1} \begin{cases}N^{\alpha-\beta} \ln \left(1+c_{2} N / h\right), & \text { if } r(\tilde{x}) \sim N^{-1} \\ N^{\alpha-\beta}, & \text { in other cases }\end{cases}
$$

holds, $c_{1}, c_{2}$ are constants non-depending on $h, N$.

Proof. Assuming $N$ is enough large so that both operators $K_{d}$ and $K_{d, N}$ are invertible in spaces $l^{2}$ and $l_{N}^{2}$ respectively let us consider the difference

$$
\begin{aligned}
& u_{d}(\tilde{x})-u_{d, N}(\tilde{x})=\left(K_{d}^{-1} v_{d}\right)(\tilde{x})-\left(K_{d, N}^{-1} v_{d, N}\right)(\tilde{x})=\left(\left(K_{d}^{-1} v_{d}\right)(\tilde{x})\right. \\
& \left.-\left(K_{d}^{-1} v_{d, N}\right)(\tilde{x})\right)+\left(\left(K_{d}^{-1} v_{d, N}\right)(\tilde{x})-\left(K_{d, N}^{-1} v_{d, N}\right)(\tilde{x})\right)=I_{1}+I_{2}
\end{aligned}
$$

where

$$
\begin{aligned}
& I_{1}=\left(K_{d}^{-1} v_{d}\right)(\tilde{x})-\left(K_{d}^{-1} v_{d, N}\right)(\tilde{x}), \\
& I_{2}=\left(K_{d}^{-1} v_{d, N}\right)(\tilde{x})-\left(K_{d, N}^{-1} v_{d, N}\right)(\tilde{x}) .
\end{aligned}
$$

We'll consider summands separately and give pointwise estimates for them. For

$$
I_{2}(\tilde{x})=\left(K_{d}^{-1} v_{d, N}\right)(\tilde{x})-\left(K_{d, N}^{-1} v_{d, N}\right)(\tilde{x})
$$

we have $I_{2}(\tilde{x})=0$ because this difference is not zero for points from $h \mathbb{Z}^{m} \backslash Q_{N}$ only, so we need to estimate $I_{1}(\tilde{x})$ only.

First we need to say some words on a structure of operators $K_{d}^{-1}$ and $K_{d, N}^{-1}$ which we have constructed for operators $K_{d}: l^{2} \rightarrow l^{2}$ and $K_{d, N}: l_{N}^{2} \rightarrow l_{N}^{2}$. Lemma 1 [19] implies that the norm of the operator $K_{d}$ doesn't depend on $h$. Also we have an analogue assertion for the operator $K_{d, N}$ (Lemma 2). Moreover the operator $K_{d}^{-1}$ is generated by Calderon-Zygmund operator with symbol $\sigma^{-1}(\xi)$ and corresponding kernel $K^{-1}(x)$, so that the kernel $K_{d}^{-1}(\tilde{x})$ of 
the discrete operator $K_{d}^{-1}$ is a restriction of the kernel $K^{-1}(x)$ on the lattice $h \mathbb{Z}^{m}$.

Further the operator $K_{d, N}^{-1}$ is constructed by the same way. We take the discrete kernel $K_{d}^{-1}(\tilde{x})$ then we take its restriction on $Q_{N}$ and a periodic continuation on a whole $h \mathbb{Z}^{m}$. A symbol of a such operator will be $\sigma_{d, N}^{-1}(\tilde{\xi})$, and we conserve all required properties.

Let us start to estimate $I_{1}(\tilde{x})$.

$$
\begin{aligned}
I_{1}(\tilde{x}) & =\sum_{\tilde{y} \in h \mathbb{Z}^{m}} K_{d}^{-1}(\tilde{x}-\tilde{y})\left[v_{d}(\tilde{y})-v_{d, N}(\tilde{y})\right] h^{m} \\
& =\sum_{\tilde{y} \in h \mathbb{Z}^{m} \backslash Q_{N}} K_{d}^{-1}(\tilde{x}-\tilde{y}) v_{d}(\tilde{y}) h^{m}
\end{aligned}
$$

and we need to estimate the last sum only.

1) $r(\tilde{x}) \sim N^{-1}$. Here taking into account that $|\tilde{x}-\tilde{y}| \geq h$ we obtain

$$
|\tilde{x}-\tilde{y}| \sim|\tilde{x}-\tilde{y}|+h .
$$

We'll represent $I_{1}(\tilde{x})$ as a sum $I_{1}(\tilde{x})=I_{11}(\tilde{x})+I_{12}(\tilde{x})$, where

$$
\begin{aligned}
& I_{11}(\tilde{x})=\sum_{\tilde{y} \in A \cap\left(h \mathbb{Z}^{m} \backslash Q_{N}\right)} K_{d}^{-1}(\tilde{x}-\tilde{y}) v_{d}(\tilde{y}) h^{m}, \\
& I_{12}(\tilde{x})=\sum_{\tilde{y} \in B \cap\left(h \mathbb{Z}^{m} \backslash Q_{N}\right)} K_{d}^{-1}(\tilde{x}-\tilde{y}) v_{d}(\tilde{y}) h^{m}
\end{aligned}
$$

and for $\tilde{y} \in A \cap\left(h \mathbb{Z}^{m} \backslash Q_{N}\right)$ we have $r(\tilde{y}) \sim N^{-1}$, for $\tilde{y} \in B \cap\left(h \mathbb{Z}^{m} \backslash Q_{N}\right)$ we assume that $r(\tilde{y}) \gg N^{-1}$. Then

$$
\left|I_{11}(\tilde{x})\right| \leq \sum_{\tilde{y} \in A \cap\left(h \mathbb{Z}^{m} \backslash Q_{N}\right)}(|\tilde{x}-\tilde{y}|+h)^{-m}(1+|\tilde{y}|)^{\alpha-\beta} h^{m}
$$

and for enough small $h$ we have

$$
\left|I_{11}(\tilde{x})\right| \leq c \int_{D_{N}(\tilde{x})}(|\tilde{x}-y|+h)^{-m}(1+|y|)^{\alpha-\beta} d y,
$$

where $D_{N}(\tilde{x})$ is a ball with a center in $\tilde{x}$ and radius $\sim N$. Using spherical coordinates with a center in $\tilde{x}$ we obtain

$$
\left|I_{11}(\tilde{x})\right| \leq c_{1} N^{\alpha-\beta} \int_{0}^{c_{2} N} \frac{d t}{t+h}
$$

and thus

$$
\left|I_{11}(\tilde{x})\right| \leq c_{1} N^{\alpha-\beta} \ln \left(1+c_{2} N / h\right) .
$$

For $I_{12}(\tilde{x})$ we use another estimate. We write

$$
\begin{aligned}
\left|I_{12}(\tilde{x})\right| & \leq \sum_{\tilde{y} \in B \cap\left(h \mathbb{Z}^{m} \backslash Q_{N}\right)}(|\tilde{x}-\tilde{y}|+h)^{-m}(1+|\tilde{y}|)^{\alpha-\beta} h^{m} \\
& =\sum_{\tilde{y} \in B \cap\left(h \mathbb{Z}^{m} \backslash Q_{N}\right) \cap\{|\tilde{y}| \leq c|\tilde{x}|\}}(|\tilde{x}-\tilde{y}|+h)^{-m}(1+|\tilde{y}|)^{\alpha-\beta} h^{m}
\end{aligned}
$$




$$
\begin{aligned}
& +\sum_{\tilde{y} \in B \cap\left(h \mathbb{Z}^{m} \backslash Q_{N}\right) \cap\{|\tilde{y}|>c|\tilde{x}|\}}(|\tilde{x}-\tilde{y}|+h)^{-m}(1+|\tilde{y}|)^{\alpha-\beta} h^{m} \\
& =I_{121}(\tilde{x})+I_{122}(\tilde{x}) .
\end{aligned}
$$

For $I_{121}(\tilde{x})$ we have $|\tilde{x}-\tilde{y}| \sim|\tilde{x}|$, and

$$
\left|I_{121}(\tilde{x})\right| \leq c|\tilde{x}|^{-m} \int_{|\tilde{x}| \leq|y| \leq c|\tilde{x}|}(1+|y|)^{\alpha-\beta} d y \sim|\tilde{x}|^{\alpha-\beta},
$$

consequently $\left|I_{121}(\tilde{x})\right| \leq c N^{\alpha-\beta}$. For $I_{122}(\tilde{x})$ we have $|\tilde{x}-\tilde{y}| \sim|\tilde{y}|$, and then

$$
\left|I_{122}(\tilde{x})\right| \leq c \int_{|y|>N}(1+|y|)^{\alpha-\beta-m} d y \sim|\tilde{N}|^{\alpha-\beta} .
$$

2) $r(\tilde{x}) \sim N$. Here we have $|\tilde{x}-\tilde{y}| \sim N$. Thus

$$
\begin{aligned}
\left|I_{1}(\tilde{x})\right| & =\left|\sum_{\tilde{y} \in h \mathbb{Z}^{m} \backslash Q_{N}} K_{d}^{-1}(\tilde{x}-\tilde{y}) v_{d}(\tilde{y}) h^{m}\right| \leq \sum_{\tilde{y} \in h \mathbb{Z}^{m} \backslash Q_{N}}\left|K_{d}^{-1}(\tilde{x}-\tilde{y})\right|\left|v_{d}(\tilde{y})\right| h^{m} \\
& \leq c N^{-m} \sum_{\tilde{y} \in h \mathbb{Z}^{m} \backslash Q_{N}}(1+|\tilde{y}|)^{\alpha-\beta} h^{m} .
\end{aligned}
$$

Since we are interested in small $h$ the last sum can be dominated by the following integral

$$
\int_{\mathbb{R}^{m} \backslash Q_{N}}|y|^{\alpha-\beta} d y
$$

and calculations with spherical coordinates give the estimate for $\beta-\alpha>0$

$$
\left|I_{1}(\tilde{x})\right| \leq c N^{\alpha-\beta} .
$$

3) $r(\tilde{x}) \sim 1$. So we have the following estimate

$$
\begin{aligned}
\left|I_{1}(\tilde{x})\right| & =\left|\sum_{\tilde{y} \in h \mathbb{Z}^{m} \backslash Q_{N}} K_{d}^{-1}(\tilde{x}-\tilde{y}) v_{d}(\tilde{y}) h^{m}\right| \leq \sum_{\tilde{y} \in h \mathbb{Z}^{m} \backslash Q_{N}}\left|K_{d}^{-1}(\tilde{x}-\tilde{y})\right|\left|v_{d}(\tilde{y})\right| h^{m} \\
& \leq c \sum_{\tilde{y} \in h \mathbb{Z}^{m} \backslash Q_{N}}(1+|\tilde{y}|)^{\alpha-\beta-m} h^{m},
\end{aligned}
$$

because $|\tilde{x}-\tilde{y}| \sim 1+|\tilde{y}|$. Since we are interested in small $h$ the last sum can be dominated by the following integral

$$
\int_{\mathbb{R}^{m} \backslash Q_{N}}|y|^{\alpha-\beta-m} d y
$$

and calculations with spherical coordinates give the estimate for $\beta-\alpha>0$

$$
\left|I_{1}(\tilde{x})\right| \leq c N^{\alpha-\beta} .
$$




\section{Conclusions}

Our considerations give a certain algorithm for solving a simplest singular integral equation in a whole space $\mathbb{R}^{m}$, and also in $\mathbb{R}_{+}^{m}$ taking into account authors' conclusions in $[21,22]$. The error estimate for finite discrete solutions shows that varying $N, h$ we can obtain a necessary sharpness. Collecting all authors' results $[18,19,20,21,22]$ related to equations (4.1), (4.2), (4.3) we conclude that there is a certain correspondence between solvability of these equations and their solutions.

\section{References}

[1] S. Abdullaev. Multidimensional singular integral equations in Holder spaces with a weight degenerating on a non-compact set. Doklady Akademii Nauk SSSR, 308(6):1289-1292, 1989.

[2] A. Böttcher and B. Silbermann. Analysis of Toeplitz Operators. Springer Monographs in Mathematics. Springer-Verlag, Berlin, 2006. https://doi.org/10.1007/3-540-32436-4.

[3] V. Didenko and B. Silbermann. Approximation of Additive Convolution-like Operators. Real $C^{*}$-algebra Approach. Birkhauser, Basel, 2008.

[4] F.D. Gakhov. Boundary Value Problems. International Series of Monographs in Pure and Applied Mathematics. Pergamon, Massachusetts, Palo Alto, London, 1966.

[5] I.C. Gohberg and I.A. Feldman. Convolution Equations and Projection Methods for Their Solution (Translations of Mathematical Monographs). The American Mathematical Society, Providence, 1974.

[6] R. Hagen, S. Roch and B. Silbermann. $C^{*}$-algebras and Numerical Analysis. Pure and applied mathematics. A series of Monographs and textbooks. Marcel Dekker, New York, 2001.

[7] I. Lifanov, L. Poltavskii and G. Vainikko. Hypersingular Integral Equations and their Applications. Chapman \& Hall/CRC, Boca Raton, 2004.

[8] I.K. Lifanov. Singular Integral Equations and Discrete Vortices. VSP, Utrecht, The Netherlands, 1996.

[9] I.K. Lifanov and L.N. Poltavskii. Pseudodifference operators and uniform convergence of divided differences. Sbornik: Mathematics, 193(2):205-230, 2002. https://doi.org/10.1070/SM2002v193n02ABEH000627.

[10] S.G. Mikhlin and S. Prössdorf. Singular Integral Operators. Springer-Verlag, Berlin, 1986.

[11] N.I. Muskhelishvili. Singular Integral Equations. Springer Netherlands, 1958. https://doi.org/10.1007/978-94-009-9994-7.

[12] A. Pedas and E. Tamme. Spline collocation method for integro-differential equations with weakly singular kernels. Journal of Computational and Applied Mathematics, 197(1):253-269, 2006. https://doi.org/10.1016/j.cam.2005.07.035.

[13] A. Pedas and E. Tamme. A discrete collocation method for Fredholm integrodifferential equations with weakly singular kernels. Applied Numerical Mathematics, 61(6):738-751, 2011. https://doi.org/10.1016/j.apnum.2011.01.006. 
[14] S. Prössdorf and B. Silbermann. Numerical Analysis for Integral and Related Operator Equations, volume 52 of Operator Theory: Advances and Applications. Birkhäuser, Basel, 1991.

[15] J. Saranen and G. Vainikko. Periodic Integral and Pseudodifferential Equations with Numerical Approximation. Springer Monographs in Mathematics. Springer, Berlin, 2002. https://doi.org/10.1007/978-3-662-04796-5.

[16] E. Vainikko and G. Vainikko. A spline product quasi-interpolation method for weakly singular Fredholm integral equations. SIAM Journal on Numerical Analysis, 46(4):1799-1820, 2008. https://doi.org/10.1137/070693308.

[17] G. Vainikko. Multidimensional Weakly Singular Integral Equations, volume 1549 of Lecture Notes in Mathematics. Springer, Berlin, Heidelberg, 1993. https://doi.org/10.1007/BFb0088979.

[18] A.V. Vasil'ev and V. B. Vasil'ev. Periodic Riemann problem and discrete convolution equations. Differential Equations, 51(5):652-660, 2015. https://doi.org/10.1134/S0012266115050080.

[19] A.V. Vasil'ev and V.B. Vasil'ev. On the solvability of certain discrete equations and related estimates of discrete operators. Doklady Mathematics, 92(2):585589, 2015. https://doi.org/10.1134/S1064562415050312.

[20] A.V. Vasilyev and V.B. Vasilyev. Numerical analysis for some singular integral equations. Neural, Parallel \& Scientific Computations, 20(3/4):313-326, 2012.

[21] A.V. Vasilyev and V.B. Vasilyev. Discrete singular operators and equations in a half-space. Azerbaijan Journal of Mathematics, 3(1):84-93, 2013.

[22] A.V. Vasilyev and V.B. Vasilyev. Discrete singular integrals in a halfspace. In V.V. Mityushev and M.V. Ruzhansky(Eds.), Current Trends in Analysis and Its Applications: Proceedings of the 9th ISAAC Congress, Kraków 2013, pp. 663-670, Cham, 2015. Springer International Publishing. https://doi.org/10.1007/978-3-319-12577-0_72. 